\title{
Ignition and Extinction Modeling of Hydrothermal Flames
}

\section{Mengmeng Ren ${ }^{a}$, Shuzhong Wang ${ }^{\mathrm{b},{ }^{*}}$ and Chuang Yang ${ }^{\mathrm{c}}$}

Key Laboratory of Thermo-Fluid Science and Engineering, Ministry of Education, School of Energy and Power Engineering, Xi'an Jiaotong University, Xi'an, Shaanxi ,710049, China

\author{
aren.meng.meng@163.com, 'szwang@aliyun.com, ' 1534596543@qq.com
}

Keywords: Supercritical water oxidation, Hydrothermal flame, Fluent, Reactive flow modeling, Ignition, Extinction

\begin{abstract}
A modeling research is conducted for a coaxial hydrothermal combustor. The single eddy-dissipation model and finite-rate vs. eddy-dissipation model are used comparably. The latter is proved to be more precise to reflect the flow-reaction feature near the nozzle and able to predict the ignition and extinction temperatures. Large discrepancy between fuel and oxygen inlet velocities results to a recirculation zone in the reactor, which plays an important role in ignition and flame stability. Since the recirculation zone decrease with the increase in fuel concentration, the ignition temperature of $60 \%$ methanol is even higher than that of $30 \%$ methanol. The predicted extinction temperature is lower than the experimental data, which may be caused by the unsuitable of the chemical reaction model from the Fluent database.
\end{abstract}

\section{Introduction}

Hydrothermal flame is the flame produced in supercritical water. It is a kind of high-pressure ultra-wet combustion, and was first conducted by Franck [1] in 1988. When temperature and pressure exceeds its critical point $\left(p_{\mathrm{c}}=22.1 \mathrm{MPa}, T_{\mathrm{C}}=374^{\circ} \mathrm{C}\right)$, water exists as a single dense phase with transport properties comparable to those of a gas and solvent properties even better than liquid water [2]. These unique properties make supercritical water a possible medium for flames to be ignited and maintained. The hydrothermal flame is mainly used in supercritical water oxidation (SCWO) as an inner heat source to solve the corrosion and plugging problems in preheating process [3]. Recent years, other utilizations of hydrothermal flame were also proposed, such as thermal spallation drilling [4] and high-pressure direct-fired steam-gas generator (HDSG) [5]. Ignition and extinction temperatures are the two most investigated characteristics upon hydrothermal flames [6, 7]. It was proved that the ignition and extinction temperatures vary with the configuration of reactors [8], actually the flow patterns. Hence studying the flow field and its effect on ignition and extinction temperatures is demanded.

\section{Method}

Two turbulent and reaction model in Fluent Software are used: eddy-dissipation model and finite-rate vs. eddy-dissipation model. The former assume the chemical reaction rate is fast relative to the transport process and the reaction rate is only dependent on the dissipation of turbulent eddies which contains the reactants [9]. The latter compares the chemical reaction rate and the eddy-dissipation reaction rate and chooses the smaller as the variation rate of reactants. This helps to capture the characteristics when chemical reaction rate is comparable with or even lower than eddy-dissipation reaction rate, especially at ignition and extinction conditions.

The steady state solution is calculated. For ignition conditions, the initial temperatures in the reactor are set the same with the inlet temperature. With this temperature reduced step by step, we check if the converged result show high-temperature zones in the reactor, which illustrating the ignition is happen. The lowest inlet temperature which can result to high-temperature zones are regarded as the ignition temperature. In the same way, the extinction temperature of a specific reactor 
configuration and methanol concentration is approached, expect for the set of initial temperatures in the reactor, which is set as the estimated flame temperature.

The reactor configuration investigated in this study is the coaxial hydrothermal burner developed in ETH[7]. Considering the axisymmetric characteristic, half of the axial section is meshed as the calculation zone, as shown in Figure 1. Methanol solution and oxygen are injected from the central orifice and the outer annulus separately.

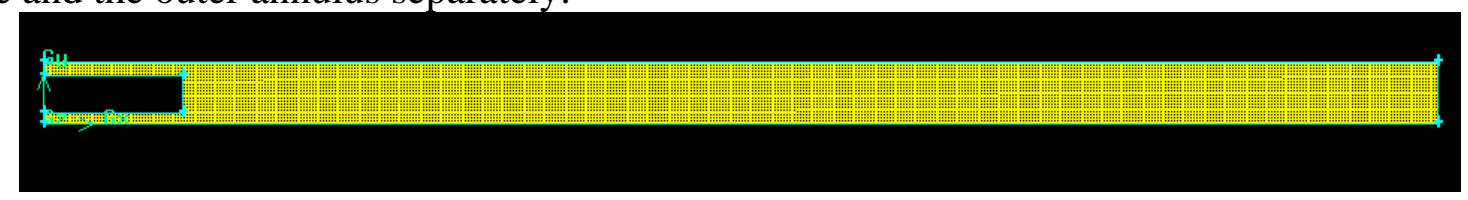

Figure 12 -D axisymmetric mesh of coaxial hydrothermal burner.

\section{Result and Discussion}

The reaction rates calculated by chemical Arrhenius equations and eddy-dissipation rate, and the smaller one among them which is used for iteration are shown in Figure 1. It shows that the turbulent reaction rate is rather high at the zones very close to the nozzle, while the chemical reaction rate rises until a certain distance beyond the nozzle. This indicates that the single eddy-dissipation model could not reflect the flow-reaction characteristic near the nozzle. Hence the finite-rate vs. eddy-dissipation model is used in the following sections.
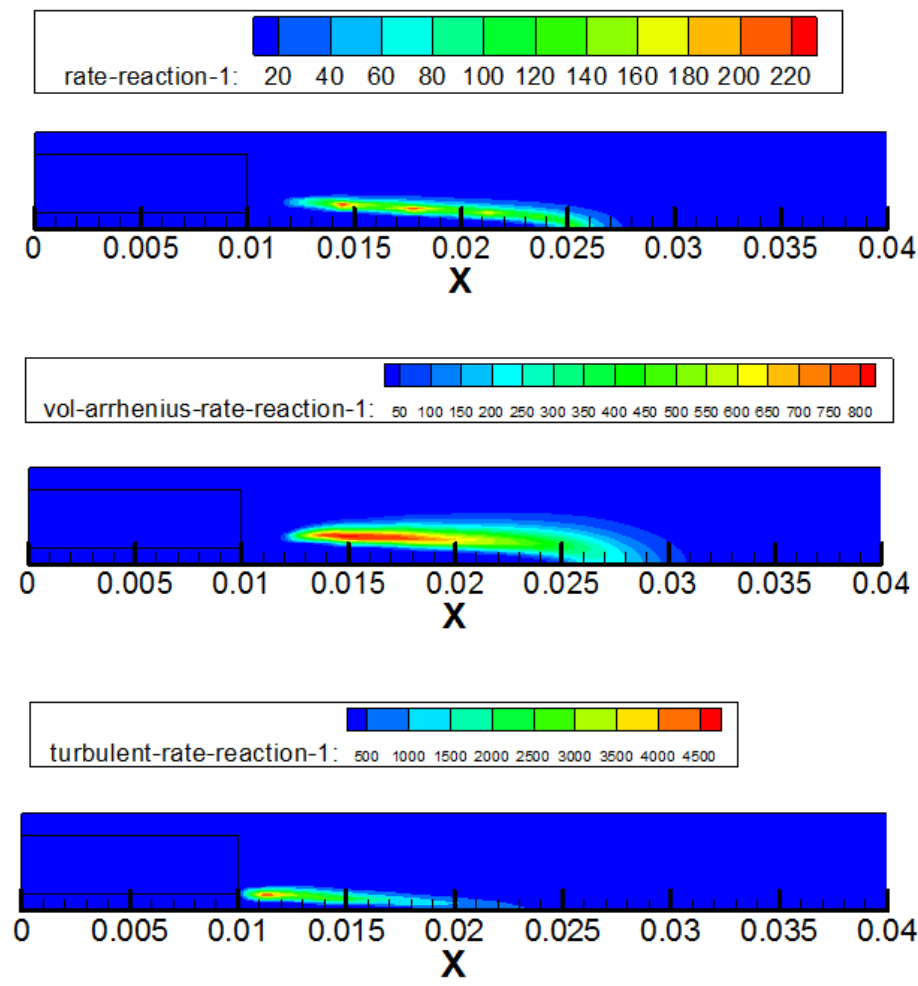

Figure 2 Reaction rates of $12 \%$ methanol solution and $700 \mathrm{~K}$ inlet temperature at ignition condition: reaction rate for iteration (upper); chemical reaction rate (middle); eddy-dissipation reaction rate (down).

The velocity fields inside the reactor at different conditions are shown in Figure 3. For 12\% methanol solution at $700 \mathrm{~K}$ inlet temperature, a large recirculation zone is shown from the result, which is caused by the large discrepancy between fuel and oxygen inlet velocity. This back-mixing flow brings the hot exhausted fluid back to heat and ignite the inlet cold reactants. With the increase in methanol concentration, the recirculation zone becomes smaller. This is because the oxygen flow rate increases and the velocity discrepancy decreases. For $60 \%$ methanol solution at $530 \mathrm{~K}$ inlet 
temperature, the recirculation zone disappears and no ignition happens. Comparing with the result from $30 \%$ methanol solution at the same $530 \mathrm{~K}$ inlet temperature, the variation of flow field lead to the ignition temperature of high concentration (60\%) becomes higher than that of low concentration (30\%).
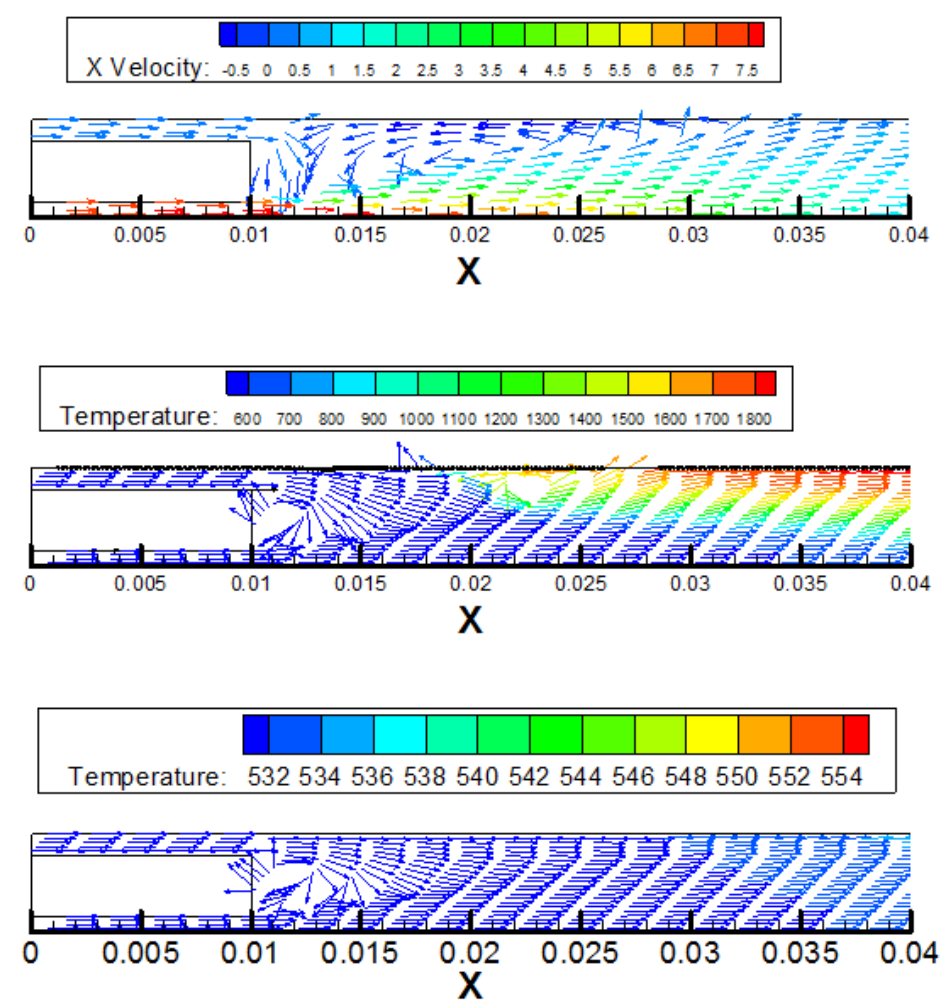

Figure 3 Calculated velocity field (all calculated for ignition conditions, equivalence ratio=1): 12\% methanol solution, inlet temperature of $700 \mathrm{~K}$ (upper); 30\% methanol solution, inlet temperature of 530K (middle); 60\% methanol solution, inlet temperature of 530K (down).

The ignition condition and extinction condition are respectively resolved for the methanol concentration of $12 \mathrm{~mol} \%$ and inlet temperature of $500 \mathrm{~K}$. The result shown high-temperature flame zone at extinction condition but not at ignition temperature, which indicates that the ignition temperature at this condition is higher than $500 \mathrm{~K}$ and its extinction is lower than $500 \mathrm{~K}$. Then we reduce the inlet temperature at extinction conditions. Until it down to 400K, no high-temperature flame was resulted. Hence $400 \mathrm{~K}$ can be regarded as the extinction temperature at this condition. However, for $12 \%$ methanol solution, the experimental extinction temperature is about 650K [7], which is much higher than this modeled value. This deviation may be caused by the unsuitable chemical Arrhenius coefficients from the Fluent database. Nevertheless, the result shows the feasibility of this method to calculate the ignition and extinction temperature, which are distinguished by the initial values' set.

\section{Conclusion and outlook}

Better than the single eddy-dissipation model, the finite-rate vs. eddy-dissipation model could reflect the reaction and flow characteristic especially near the nozzle more precisely and can be used to predict the ignition and extinction temperatures at different conditions. The recirculation zone induced by the large velocity discrepancy is the main flame-stable regime, which varies from different methanol concentration at specific reactor configurations. Hence the reactor configuration should be design for the desired operation conditions specially. The unsuitable chemical Arrhenius coefficients from the Fluent database causes the deviation of calculated ignition and extinction 
temperatures. Further research should import more suitable chemical reaction model, such as the elementary reaction model developed by our parallel work.
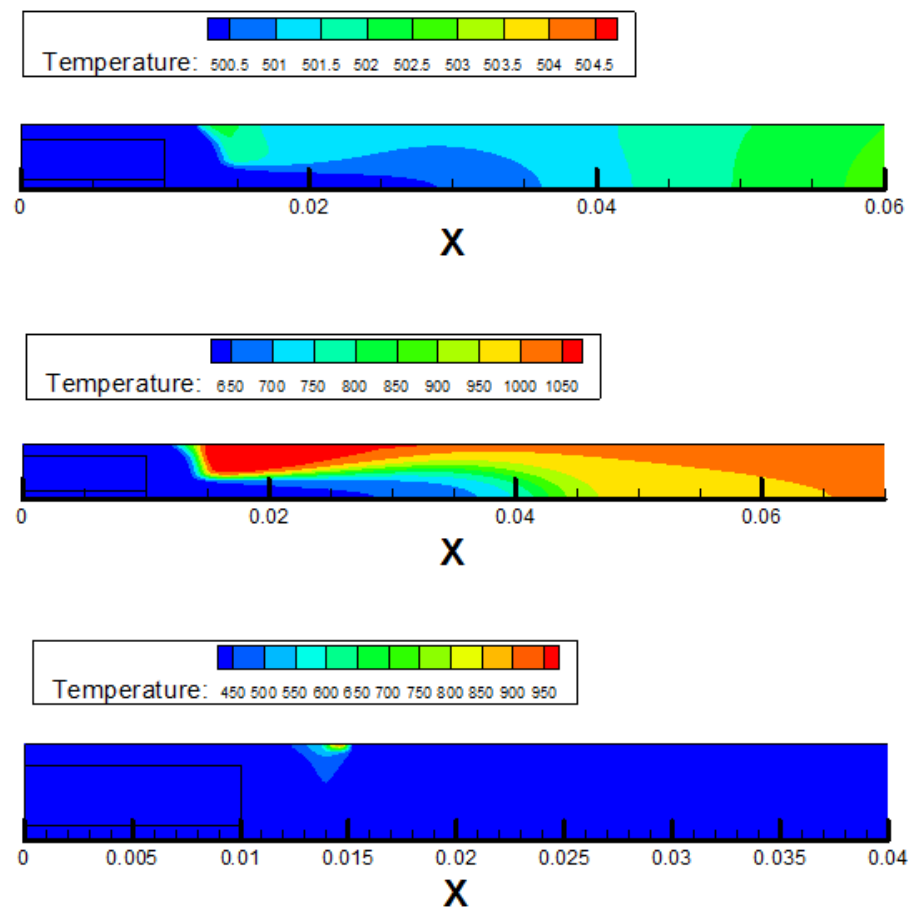

Figure 4 Temperature distributions in reactor for $12 \%$ methanol solution: inlet temperature of $500 \mathrm{~K}$ at ignition conditions (upper); inlet temperature of $500 \mathrm{~K}$ at extinction conditions (middle); inlet temperature of $400 \mathrm{~K}$ at extinction conditions (down).

\section{Acknowledgements}

This work is supported by the Projects from National Natural Science Foundation of China (No. 21576219, No. 51406146).

\section{Reference}

[1] Kruse, A. and H. Schmieder, Supercritical oxidation in water and carbon dioxide. Environmental progress, 17 (1998) 234-239.

[2] Brunner, G., Near and supercritical water. Part II: Oxidative processes. Journal of Supercritical Fluids, 47(2009) 382-390.

[3] Augustine, C. and J.W. Tester, Hydrothermal flames: From phenomenological experimental demonstrations to quantitative understanding. The Journal of Supercritical Fluids, 47 (2009) 415-430.

[4] Stathopoulos, P., T. Meier, and P.R. von Rohr, Hydrothermal flame impingement experiments. Combustion chamber design and impingement temperature profiles. Journal of Supercritical Fluids, 89(2014) 48-57.

[5] Ren, M.M., et al., High-pressure direct-fired steam-gas generator (HDSG) for heavy oil recovery. Applied Mechanics and Materials, 577(2014) 523-526.

[6] Steeper, R., et al., Methane and methanol diffusion flames in supercritical water. The Journal of Supercritical Fluids, 5(1992) 262-268.

[7] Wellig, B., et al., Hydrothermal methanol diffusion flame as internal heat source in a SCWO reactor. The Journal of Supercritical Fluids, 49(2009) 59-70. 
[8] Príkopský, K., Characterization of continuous diffusion flames in supercritical water, Doctor of Technical Sciences Thesis. 2007, Swiss Federal Institute of Technology.

[9] Narayanan, C., et al., Numerical modelling of a supercritical water oxidation reactor containing a hydrothermal flame. The Journal of Supercritical Fluids, 2008. 46(2): p. 149-155. 